[Aus dem Institut für Infectionskrankheiten in Berlin.]

\title{
Ist die Virulenz der Cholerabacillen abhängig von ihrer Giftigkeit? ${ }^{1}$
}

Von

Dr. Freiherrn von Dungern.

Die Virulenz der Cholerabacillen kann eine recht verschiedene sein. Frisch aus dem Darme Cholerakranker gezüchtete Vibrionen können schon, in geringer Menge in's Peritoneum von Meerschweinchen eingeführt, sich am Leben erhalten und vermehren. ${ }^{2}$ Lange auf künstlichen Nährböden fortgezüchtete Culturen sind nur dann im Stande, unter den gleichen Bedingungen ein parasitäres Leben zu führen, wenn sie in grossen Dosen eingebracht werden. Worauf beruht nun dieser Unterschied? Ist derselbe bedingt durch eine verschiedene Widerstandskraft der virulenten und nicht virulenten Vibrionen den baktericiden Stoffen des Organismus gegenüber, oder zeigt er sich abhängig von der Giftigkeit der Cultur, welche die baktericiden Schutzkräfte unterdrückt? Diese Frage konnte durch einen genauen Vergleich der Giftwirkung virulenter und nicht virulenter Vibrionen entschieden werden.

$\mathrm{Zu}$ den Versuchen benutzte ich einerseits eine mir von Hrn. Prof. Pfeiffer. übergebene Cultur, welche bei einem Cholerafall der letzten Epidemie in Ostpreussen isolirt worden ist. Die Virulenz derselben war so gross, dass $1 / 8$ Oese $\left(1 / 4{ }^{\mathrm{mg}}\right)$ einer 20 Stunden alten Agarcultur ein Meerschweinchen von $200 \mathrm{grm}$ tödtete. Im Verlauf der Untersuchung nahm die Virulenz etwas $a b$, so dass nur noch $1 /$ Oese unter gleichen Bedingungen tödtete; sie wurde dann wieder durch Meerschweinchenpassagen verstärkt. Als nicht virulente Form diente mir eine Cultur, welche ursprünglich aus Calcutta stammt und seit 8 Jahren im Institut für

1. Eingegangen am 6. April 1895.

${ }^{2}$ R. Pfeiffer, Stadien über Choleraätiologie. Diese Zeitschrift. Bd. XVI. 
Infectionskrankheiten fortgezüchtet wird. Diese Vibrionen wurden aus dem Darme eines Cholerakranken gezüchtet und sind auch nach den Immunisirungsversuchen von Prof. R. Pfeiffer als echte Cholerabacillen anzusehen. Die Virulenz derselben ist fast vollständig verloren gegangen; nach peritonealer Injection selbst ganz grosser Mengen (10 oder $20^{\mathrm{mg}}$ ), denen die Meerschweinchen erliegen, kommt es doch zur Abtödtung fast sämmtlicher eingeführten Mikroorganismen.

Es wurde darauf gesehen, dass immer unter möglichst gleichen Versuchsbedingungen gearbeitet wurde. Es handelte sich immer um Agarculturen, welche 20 Stunden bei $37^{\circ}$ gewachsen waren. Die Abtödtung der Kommabacillen geschah theilweise durch Chloroformdämpfe, welche jedesmal $1 / 2$ Stunde im Brütschrank einwirkten.

Die Cultur wird dadurch in eine zähe Masse umgewaudelt. Von dieser wurde jedesmal eine bestimmte Menge abgewogen, in $1^{\text {com }}$ Bouillon möglichst fein suspendirt und einem Meerschweinchen von etwa $200 \mathrm{grm}$ Gewicht in die Bauchhöhle injicirt. Eine Verletzung des Darmes wurde nach Möglichkeit vermieden. ${ }^{1}$ Es zeigte sich bei diesen Versuchen, dass die Thiere unter diesen Bedingungen nicht gleichmässig reagiren, und dass auch die kleinste tödtliche Dose keine ganz constante ist. Ein wesentlicher Unterschied in der Giftwirkung der Cholera Ostpreussen und Cholera Calcutta konnte jedoch nicht constatirt werden.

\section{Cholera Ostpreussen.}

Meerschweinchen, $210 \mathrm{grm}, 37.4^{\circ}$, erhält $1^{1 / 2}$ Uhr $10^{\mathrm{mg} .} 3 \mathrm{Uhr} 37 \cdot 2^{0}$; $8 \mathrm{Uhr} 28 \cdot 0^{\circ} ;+$ in der Nacht.

In der Bauchhöhle klares Exsudat (steril). Auf der Leber leichte fibrinöse Auflagerungen, Organe normal.

Meerschweinchen, $225^{\mathrm{grm}}, 37: 2^{\circ}$, erhält 2 Uhr $10^{\mathrm{mg} .} 7 \mathrm{Uhr} 34 \cdot 5^{0}$; nach 22 Stunden $37 \cdot 7^{\circ}$.

Meerschweinchen, $185 \mathrm{grm}, 37.2^{0}$, erhält 12 Uhr $20^{\mathrm{mg}}$. 2 Uhr $35 \cdot 7^{\circ}$; $8 \mathrm{Uhr}+$. In der Bauchhöhle klare Flüssigkeit. Auf der Leber Auflagerungen (steril). Organe normal.

Meerschweinchen, $245^{\mathrm{grm}}, 37 \cdot 4^{0}$, erhält $12 \mathrm{Uhr} 18^{\mathrm{mg}}$. $8 \mathrm{Uhr} 34 \cdot 5^{\circ}$, nach 22 Stunden $36 \cdot 0^{\circ}$.
Cholera Calcutta.

Meerschweinchen, $205^{\mathrm{grm}}, 37 \cdot 6^{\circ}$, erhält $12^{1} / \mathrm{T}$ Uhr $10^{\mathrm{mg} .} 8 \mathrm{Uhr} 35.4^{0}$; nach 22 Stunden $37 \cdot 6 "$ ".

Meerschweinchen, $195^{\mathrm{krm}}, 38 \cdot 2^{\circ}$. erhält 1 Uhr $20^{\mathrm{mg}} .8 \mathrm{Uhr} 31.4^{0}$, sehr krank; nach 22 Stunden $37.7^{\circ}$.

Meerschweinchen, $200 \mathrm{grm}, 37 \cdot 9^{\circ}$, erhält $12^{1} / 2 \mathrm{Uhr} 20^{\mathrm{mg}}$. $8 \mathrm{Uhr} 31 \cdot 0^{0}$, sehr krank; nach 22 Stunden $38 \cdot 0^{\circ}$.

${ }^{1}$ Dr. Kolle, Beiträge zu den experimentellen Cholerastudien an Meerschweinchen. Diese Zeitschrift. Bd. XVI. 
Um constantere Resultate zu erhalten, wurden die Versuchsbedingungen etwas abgeändert. Die Verschiedenheit im Wassergehalt der Culturen wurde dadurch vermieden, dass dieselben in grösseren Mengen am gleichen Tage auf demselben Agar angelegt werden. $40^{\mathrm{mg}}$ wurden dann in $0.5^{\mathrm{ccm}}$ Bowillon suspendirt und in zugeschmolzene Glasröhrchen eingeschlossen.

Die Abtödtung sowohl der virulenten wie der nicht virulenten Vibrionen geschah in demselben Wasserbade, welches 1 Stunde lang auf 54 bis $55^{\circ}$ oder $57^{\circ}$ gehalten wurde.

Da die Resorption der Giftstoffe vom Peritoneum aus eine verschiedene sein kann, so injicirte ich dieselben auch direct in die Blutbahn. Ich wählte dazu die Vena femoralis oder V. jugularis. Die dosirte Menge wurde immer in $\mathbf{0 . 2} \mathbf{c m}$ Bouillon suspendirt mit einer fein ausgezogenen Glaspipette eingebracht. Die Thiere wurden dabei meist leicht chloroformirt. Ein Controlthier, dem ebenso in der Chloroformnarcose $0.2^{\mathrm{ccm}}$ Bouillon in die Vena femoralis injicirt wurden, zeigte darauf folgende Temperaturerniedrigung:

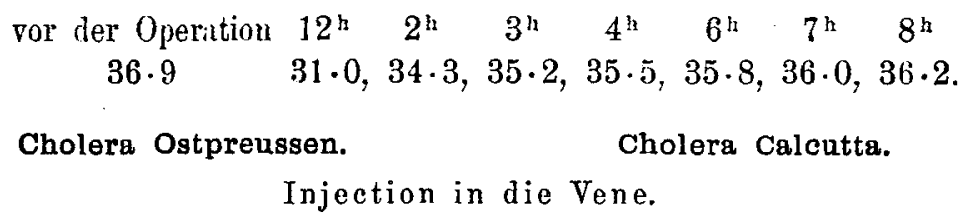

Injection in die Vene.

Meersehweinchen, $198^{\mathrm{grm}}, 37 \cdot 6^{\circ}$, erhält um $12 \mathrm{Uhr}$ in der Chloroformnarcose $8^{\text {mg }}$ Cholera Ostpreussen (eine Stunde bei $57^{\circ}$ abgetödtet) in $0.2^{\mathrm{ecm}}$ Bouillon in die Vena jugularis injicirt.

$\begin{array}{cccc}1^{\mathrm{h}} & 2^{\mathrm{h}} & 3^{\mathrm{h}} & 4^{\mathrm{h}} \\ 32 \cdot 8 & 33 \cdot 5 & 33 \cdot 7 & 34 \cdot 0 \\ 5^{\mathrm{h}} & 6^{\mathrm{h}} & 7^{\mathrm{h}} & 8^{\mathrm{h}} \\ 34 \cdot 0 & 33 \cdot 9 & 33 \cdot 9 & 33 \cdot 0\end{array}$

t in der Nacht. Kleine Hämorrhagien in den Lungen. Sonst keine Veränderungen.

Meerschweinchen, $200 \mathrm{grm}, 37 \cdot 6^{\circ}$, erhält um $12 \mathrm{Vhr}$ in der Chloroformnarcose $8^{\mathrm{mg}}$ Cholera Ostpreussen (eine Stunde bei $57^{\circ}$ abgetödtet) in $0.2 \mathrm{ccm}$ Bouillon in die Vena femoralis injicirt.

$$
\begin{array}{ccc}
\mathbf{1}^{\mathrm{h}} & \mathbf{5}^{\mathrm{h}} & \mathbf{7}^{\mathrm{h}} \\
34.5 & \mathbf{3 0 . 2} & \dagger^{+}
\end{array}
$$

Im Peritoneum etwas klare Flüssigkeit. Organe normal.
Meerschweinchen, $220 \mathrm{grm}, 38 \cdot 0^{\circ}$, erhält um 11 Uhr in der Chloroformnarcose 8 mg Cholera Calcutta (eine Stunde bei $57^{\circ}$ abgetödtet) in $0.2 \mathrm{cem}$ Bouillon in die Vena jugularis injicirt.

$$
\begin{array}{ccc}
11 / 2{ }^{\mathrm{h}} & 31 / 2^{\mathrm{h}} & 6^{1 / 2}{ }^{\mathrm{h}} \\
32 \cdot 4 & 30 \cdot 0 & \dagger
\end{array}
$$

Im Peritoneum klares Exsudat. Organe normal.

Meerschweinchen, $240 \mathrm{grm}, 36 \cdot 2^{0}$, erhält um $12 \mathrm{Uhr}$ in der Chloroformnarcose $8 \mathrm{mg}$ Cholera Calcutta (eine Stunde bei $57^{\circ}$ abgetödtet) in $0.2^{\mathrm{ccm}}$ Bouillon in die Vena femoralis injicirt. $2^{\text {h }} \quad 3^{\text {h }} \quad 4^{\text {h }} \quad 5^{\text {h }} \quad 6^{\text {h }} \quad 7^{\text {h }} 9^{\text {h }}$ $34.534 .830 .030 .029 .029 .0+$ In der Bauchhöhle klares Exsudat. Organe normal. 
Cholera Ostpreussen.

Cholera Calcutta.

Injection in die Vene.

Meerschweinchen, $210 \mathrm{grm}, 37 \cdot 2^{0}$, erhält nicht chloroformirt um $12 \mathrm{Uhr}$

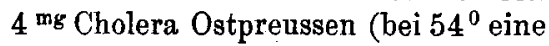
Stunde abgetödtet) in $0.2 \mathrm{~cm}$ Bouillon in die Vena jugularis injicirt.

$\begin{array}{cccc}\mathbf{1}^{\mathrm{h}} & 2^{\mathrm{h}} & 3^{\mathrm{h}} & 4^{\mathrm{h}} \\ \mathbf{3 7 \cdot 2} & \mathbf{3 8 \cdot 0} & \mathbf{3 8 \cdot 4} & 37 \cdot 9 \\ 5^{\mathrm{h}} & 6^{\mathrm{h}} & 7^{\mathrm{h}} \cdot \\ \mathbf{3 8 \cdot 0} & \mathbf{3 8 \cdot 5} & 38 \cdot 5\end{array}$

nach 20 Stunden $37 \cdot 0^{\circ}$, munter.

\section{Injection in die Bauchhöhle.}

Meerschweinchen, $187 \mathrm{grm}, 37 \cdot 0^{\circ}$, erhält $11 \mathrm{Uhr} 10 \mathrm{mg}$ Cholera Ostpreussen (eine Std. bei $57^{\circ}$ abgetödtet) in die Bauchhöhle injicirt.

$$
\begin{array}{cccc}
12^{1 / 2^{\mathrm{h}}} & 2^{\mathrm{h}} & 3^{\mathrm{h}} & 4^{\mathrm{h}} \\
37^{\circ} & 37 \cdot 3 & 37 \cdot 4 & 37^{\circ} \cdot 5 \\
5^{\mathrm{h}} & 6^{\mathrm{h}} & 7^{\mathrm{h}} & 8^{\mathrm{h}} \\
35 \cdot 5 & 35 \cdot 8 & 34 \cdot 5 & 32 \cdot 5
\end{array}
$$

nach 24 Stunden $38 \cdot 0^{\circ}$.

Durch Nackenschlag getödtet: In der Bauchhöhle wenig Exsudat mit vielen Leukocyten, keine Mikrourganismen. Auf der Leber Auflagerungen.

Meerschweinchen, $220 \mathrm{grm}, 37 \cdot 1^{0}$, erhält $1 \mathrm{Uhr} 10^{\mathrm{mg}}$ Cholera Ostpreussen (bei $54^{\circ}$ getödtet) in die Bauchhöhle eingespritzt.

$\begin{array}{cccc}2^{\mathrm{h}} & 3^{\mathrm{h}} & 4^{\mathrm{h}} & 5^{\mathrm{h}} \\ 37^{\circ} \cdot 2 & 39 \cdot 5 & 39 \cdot 3 & 38 \cdot 0 \\ 6^{\mathrm{h}} & 7^{\mathrm{h}} & 8^{\mathrm{h}} \\ 37 \cdot 6 & 38 \cdot 0 & 37 \cdot 9\end{array}$

Nach 24 Stunden getödtet: Im Peritoneum leichte Hämorrhagieen. In der Bauchhöhle wenig Exsudat mit vielen Leukocyten, keine Mikroorganismen.
Meerschweinchen, $180^{\mathrm{grm}}, 37 \cdot 1^{\circ}$, erhält 12 Uhr.10 mg Cholera Calcutta (bei $54^{0}$ getödtet) in die Bauchhöhle eingespritzt.

$\begin{array}{cccc}1^{\mathrm{h}} & 2^{\mathrm{h}} & 3^{\mathrm{h}} & 4^{\mathrm{h}} \\ 35 \cdot 0 & 35 \cdot 0 & 36 \cdot 6 & 36 \cdot 3 \\ 5^{\mathrm{h}} & 6^{\mathrm{h}} & 7^{\mathrm{h}} & 8^{\mathrm{h}} \\ 35 \cdot 0 & 33 \cdot 4 & 32 \cdot 5 & 32 \cdot 5\end{array}$

nach 24 Stunden 37.0.

Durch Nackenschlag getödtet: In der Bauchhöhle wenjg Exsudat mit vielen Leukocyten, keine Mikroorganismen.

Meerschweinchen, $195^{\mathrm{grm}}, 37.5^{\circ}$, erhält $11^{1 / 2}$ Uhr $10^{\mathrm{mg}}$ Cholera Calcutta (bei $57^{\circ}$ getödtet) in die Bauchhöhle eingespritzt.

$\begin{array}{ccccc}12^{\mathrm{h}} & 1^{\mathrm{h}} & 2^{\mathrm{h}} & 3^{\mathrm{h}} \\ 37^{2} \cdot 5 & 38 \cdot 3 & 38 \cdot 9 & 39 \cdot 4 \\ 4^{\mathrm{h}} & 5^{\mathrm{h}} & 7^{\mathrm{h}} & 8^{\mathrm{h}} & 9^{\mathrm{h}} \\ 37 \cdot 5 & 36 \cdot 5 & 36 \cdot 0 & 36 \cdot 2 & 37^{\circ} \cdot\end{array}$

nach 24 Stunden $37 \cdot 6^{\circ}$; getödtet. Auf der Leber leichte Auflagerungen. In der Bauchhöhle etwas Exsudat mit vielen Leukocyten. 


\section{Cholera Ostpreussen.}

Meersehweinchen, $260^{\mathrm{grm}}, 37 \cdot 3^{\circ}$,

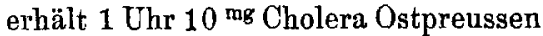
(bei $54^{\circ}$ getödtet) in die Bauchhöhle eingespritzt.

$$
\begin{array}{cccc}
2^{\mathrm{h}} & 3^{\mathrm{h}} & 4^{\mathrm{h}} & 5^{\mathrm{h}} \\
38 \cdot 6 & 38 \cdot 5 & 38 \cdot 0 & 37 \cdot 6 \\
6^{\mathrm{h}} & 7^{\mathrm{h}} & 8^{\mathrm{h}} \\
36.9 & 37 \cdot 3 & 37 \cdot 6
\end{array}
$$

Nach 24 Stunden getödtet. In der Bauchhöhle etwas Exsudat mit vielen Leukocyten, keinen Mikroorganismen.

Meerschweinchen, $220^{\mathrm{grm}}, 38 \cdot 0^{\circ}$, erhält $121 / 2$ Uhr 16 mg Chol. Ostpreussen (bei $54^{0}$ getödtet) in die Bauchhöhle.

\section{Cholera Calcutta.}

Meerschweinchen, $235^{\mathrm{grm}}, 37.9^{\circ}$, erhält 12 Uhr $10 \mathrm{mg}$ Cholera Calcutta (bei $54^{0}$ getödtet) in die Bauchhöhle.

$\begin{array}{cccc}1^{\mathrm{h}} & 2^{\mathrm{h}} & 3^{\mathrm{h}} & 4^{\mathrm{h}} \\ 37^{\mathrm{h}} \cdot 6 & 38 \cdot 1 & 39 \cdot 1 & 38^{-7} \\ 5^{\mathrm{h}} & 6^{\mathrm{h}} & 7^{\mathrm{h}} & 8^{\mathrm{h}} \\ 36 \cdot 7 & 36 \cdot 2 & 37 \cdot 2 & 37 \cdot 5\end{array}$

Nach 24 Stunden getödtet $\left(37 \cdot 7^{\circ}\right)$. In der Bauchhöhle etwas eitriges Exsudat.

Meerschweinchen, $180 \mathrm{grm}, 37 \cdot 2^{\circ}$, erhält $12 \mathrm{Uhr} 10 \mathrm{mg}$ Cholera Calcutta (bei $54^{\circ}$ getödtet) in die Bauchhöhle.

$\begin{array}{cccc}1^{\mathrm{h}} & 2^{\mathrm{h}} & 3^{\mathrm{h}} & 4^{\mathrm{h}} \\ 36 \cdot 0 & 38 \cdot 1 & \mathbf{3 8 \cdot 3} & 37^{\circ} \cdot 0 \\ 5^{\mathrm{h}} & 6^{\mathrm{h}} & 7^{\mathrm{h}} & 8^{\mathrm{h}} \\ 33 \cdot 8 & 33 \cdot 4 & 32 \cdot 6 & 32 \cdot 0\end{array}$

nach 24 Stunden $37 \cdot 6^{\circ}$; getödtet. In der Bauchhöhle viel Exsudat mit vielen Leukocyten, keine Mikroorganismen.

Meerschweinchen, $195 \mathrm{grm}, 37 \cdot 4^{\circ}$, erhält 12 Uhr $1.0 \mathrm{mg}$ Cholera Calcutta (bei $57^{\circ}$ getödtet) in die Bauchhöhle.

$$
\begin{array}{ccccc}
3^{\mathrm{h}} & 4^{\mathrm{h}} & 5^{\mathrm{h}} & 6^{\mathrm{h}} & 7^{\mathrm{h}} \\
38 \cdot 0 & 36.0 & 35 \cdot 0 & 34.9 & 34.9
\end{array}
$$

nach 24 Stunden $37 \cdot 5^{\circ}$; getödtet. Auf der Leber leichte Auflagerungen.

Meerschweinchen, $210^{\mathrm{grm}}, 37 \cdot 5^{\mathrm{D}}$, erhält $12^{1} / 2$ Uhr $16^{\mathrm{mg}}$ Cholera Calcutta (bei $54^{\circ}$ getödtet) in die Bauchhöhle.

$\begin{array}{cccc}1^{\mathrm{h}} & 2^{\mathrm{h}} & 3^{\mathrm{h}} & 4^{\mathrm{h}} \\ 37 \cdot 0 & 38 \cdot 0 & 38 \cdot 5 & 36 \cdot 7 \\ 5^{\mathrm{h}} & 6^{\mathrm{h}} & 7^{\mathrm{h}} \\ 36 \cdot 7 & 36 \cdot 4 & 35 \cdot 1\end{array}$

nach 22 Stunden $38 \cdot 0^{\circ}$; getödtet. In der Bauchhöhle Exsudat mit vielen Leukocyten, keinen Mikroorganismen.

In der Bauchhöhle Exsudat mit vielen Leukocyten, keinen Mikroorganismen. 


\section{Cholera Ostpreussen.}

Meerschweinehen, $193 \mathrm{grm}, 37 \cdot 0^{\circ}$, erhält 11 Uhr $20 \mathrm{mg}$ Chol. Ostpreussen (bei $54^{0}$ getödtet) in. die Bauchhöhle.

$\begin{array}{cccc}12^{1} /_{2}^{\mathrm{h}} & 2^{\mathrm{h}} & 3^{\mathrm{h}} & 4^{\mathrm{h}} \\ 36 \cdot 7 & 35 \cdot 5 & 35 \cdot 0 & 35 \cdot 0 \\ 5^{\mathrm{h}} & 6^{\mathrm{h}} & 7^{\mathrm{h}} & 8^{\mathrm{h}} \\ 34.9 & 34 \cdot 9 & 35 \cdot 0 & \mathbf{3 4 \cdot 0}\end{array}$

Nach 24 Stunden getödtet. In der Bauchhöhle etwas Exsudat mit vielen Leukocyten. Auf der Leber Auflagerungen.

Es zeigt sich demnach in diesen Versuchen, dass die Giftigkeit auch der virulenten Vibrionen in den hier untersuchten Choleraculturen mit Ausnahme der bereits oben besprochenen Fälle eine etwas geringere ist, als es nach den von $R$. Pfeiffer und A. Wasserman $\mathbf{n}^{1}$ angegebenen Werthen zu erwarten war. Es dürfte sich dieser Unterschied am ungezwungensten durch die verschiedenartige Abtödtung, vielleicht auch durch einen grösseren Wassergehalt der hier benutzten Culturen erklären lassen. Zur Iösung - der hier gestellten Frage kam es ja hauptsächlich darauf an, das Choleragift aus virulenten und nicht virulenten Kommabacillen unter genau den gleichen Bedingungen darzustellen. Dann können auch noch dadurch Verschiedenheiten bedingt werden, dass die Thiere nicht gleichmässig reagiren. So ersehen wir aus den Versuchen, dass genau die gleiche Giftdose bei einem Theile der Fälle eine mehr oder weniger ausgesprochene Temperaturerhöhung zur Folge hatte, bei anderen Thieren dagegen wieder eine mehr oder weniger starke Temperaturerniedrigung hervorrief. Berücksichtigen wir aber diese Schwankungen beim Vergleich der Giftwirkung beider Cholerastämme, so finden wir eine fast rollständige Uebereinstimmung. Die Giftigkeit der virulenten Cholera Ostpreussen ist nicht grösser als die der nicht virulenten Cholera Calcutta.

Da die Cholerabacillen nach der Angabe von Kolle ${ }^{2}$ nach Injection in die Blntbahn (Carotis von Meerschweinchen) sehr bald fast sämmtlich abgetödtet werden und Vergiftungserscheinungen machen, so stellte ich anch einige Versuche mit lebenden Vibrionen von Choleril Ostpreussen und Cholera Calcutta an.

Bd. XIV.

'Untersuchungen über das Wesen der Choleraimmunität. Diese Zeitschrift.

${ }^{2}$ Beiträge zu den experimentellen Cholerastndien an Meerschweinchen. Diese Zeitschrift. Bd. XVI. 
Dieselben wurden jedoch nicht in die Carotis, sondern ebenso wie die abgetödteten Culturen in die Vene eingeführt. Es zeigte sich hier ein deutlicher Unterschied. Cholera Calcutta rief in einer Dose von $\mathbf{2}^{\mathrm{mg}}$, welche bei Cholera Ostpreussen schon tödtlich war, nur eine vorübergehende Schwankung der Temperatur hervor.

Meerschweinchen, $200 \mathrm{grm}, 37 \cdot 2^{\mathrm{n}}$, erhält um $12 \mathrm{Uhr} 2 \mathrm{mg}$ Cholera Ostpreussen in die Jugularvene injicirt.

$\begin{array}{ccccccc}1^{\mathrm{h}} & 2^{\mathrm{h}} & 3^{\mathrm{h}} & 4^{\mathrm{h}} & 5^{\mathrm{h}} & 6^{\mathrm{h}} & 7^{\mathrm{h}} \\ 36 \cdot 0 & 35 \cdot 2 & 34 \cdot 5 & 34 \cdot 2 & 34 \cdot 2 & 33 \cdot 9 & 33.9\end{array}$

$\dagger$ in der Nacht. In der Bauchhöhle hämorrhagisches Exsudat. Organe normal. Aus Blut, Leber, Milz, Peritonealexsudat wachsen sehr reiche Choleraculturen.

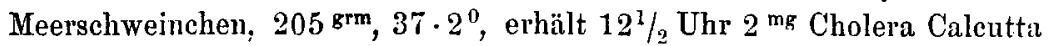
in die Jugularvene.

\begin{tabular}{|c|c|c|c|c|c|}
\hline $1^{\mathrm{h}}$ & $2^{h}$ & $3^{\mathrm{h}}$ & $4^{\mathrm{h}}$ & $5^{\text {ht }}$ & $6^{\mathrm{h}}$ \\
\hline $35 \cdot 0$ & $34 \cdot 9$ & $37 \cdot 1$ & $37 \cdot 1$ & 37.1 & 38.0 \\
\hline
\end{tabular}

nach 20 Stunden $37 \cdot 2^{0}$, munter.

Meerschweinchen, $215^{\mathrm{grm}}, 37 \cdot 2^{\circ}$, erhält um $12 \mathrm{Uhr} 2^{\mathrm{mg}}$ Cholera Calcutta in die Jugularvene injicirt.

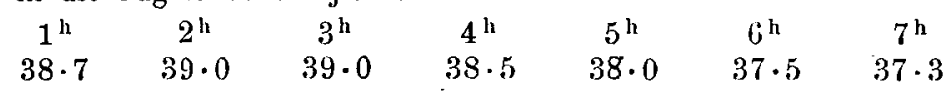

nach 20 Stunden $37 \cdot 5^{\prime \prime}$, munter.

Diese verschicdene Wirkung erklärt sich dadurch, dass $2^{\mathrm{mg}}$ bei Injection in die Vene noch nicht die tödtliche Giftdose darstellen, die virulenten Vibrionen aber nicht vollständig abgetödtet werden. Der Widerspruch mit dem bisher gewonnenen Resultate ist also nur ein scheinbarer. Wir kōnnen demnach aus den Versuchen schliessen, dass die Virulenz der Cholerabacillen vollstāndig unabhängig von ihrer Giftigkeit sein kann.

Dieses Ergebniss der Untersuchung steht in vollstïndiger Uebereinstimmung mit den Anschauungen, welche Prof. Pfeiffer in seinen Arbeiten über das Choleragift ausgesprochen hat. Für das mir gezeigte Fntgegenkommen gebührt ihm mein aufrichtiger Dank. 